\title{
Applications of Thin-Film Standards in Analytical Electron Microscopy
}

\author{
M. Watanabe* \\ * Dept of Materials Science and Engineering, Lehigh University, Bethlehem. PA 18015.
}

There are two major applications of standards in analysis: one is to evaluate analytical performance in an instrument including calibrations and other application is for quantitative analysis. For those purposes, several standards in thin-film form have been proposed and applied for X-ray energy-dispersive spectrometry (XEDS) in analytical electron microscopy (AEM).

For the first purpose to characterize the analytical performance of an instrument, Egerton's NiOx test specimen has widely been used [1-3]. By taking a single XEDS spectrum from the NiOx test specimen, detector-performance parameters, such as the energy resolution, peak-to-background ratio, detector collection angle, etc., can be determined. The system energy resolution defined at $5.9 \mathrm{keV}$ is converted from the full-width at half-maximum value of the $\mathrm{Ni} \mathrm{K} \alpha$ peak in the spectrum from NiOx, as proposed by Bennett and Egerton [2]. The energy resolution of XEDS system is the major parameter can be controlled by a selection of the detector process time. In other words, the energy resolution varies depending on the process time setting, as shown in Fig. 1(a). The system energy resolution should be known before performing X-ray analysis. The P/B ratio and $\mathrm{IHC}$, which can also be determined from a single spectrum of $\mathrm{NiOx}$, are useful to evaluate the AEM-XEDS interface [4]. Furthermore, degradation of detector performance due to accumulated contamination and ice at the detector window can be monitored by determining thicknesses of those layers from two spectra acquired at different time period [3]. To perform these measurements routinely and consistently, a set of plug-ins for Gatan DigitalMicrograph named XUtils has been developed by the author [5] and is freely available from the author's web site [6]. Since the $\mathrm{NiOx}$ test specimen is no longer available, a similar $\mathrm{NiO}$ test specimen (NiOxL) is now fabricated at Lehigh as well. The microstructure of the NiOxL test specimen and an XEDS spectrum from the NiOxL are shown in Fig. 1(b) and (c), respectively.

For quantitative analysis by XEDS in AEM, well-characterize standard specimens are required, i.e. not only composition but also thickness of the film must be known. There is only one standard to satisfy the conditions above, that is the NIST standard reference material (SRM) 2063/2063a. This thin film contains $\mathrm{Mg}, \mathrm{Si}, \mathrm{Ca}, \mathrm{Fe}$ and $\mathrm{O}$ and has the advantage that its composition is known to a high degree of accuracy, as are its thickness and density. Although the SRM 2063/2063a thin film was originally designed for the $k$-factor determination [7], this standard is ideal for the $\zeta$-factor determination in $\zeta$-factor method (which offers quantification not only of composition but also of specimen thickness proposed by the author) [8]. In this determination process, $\zeta$-factors of elements (which are not contained in the SRM2063/2063a standard) can also be estimated from a single spectrum from the SRM2063/2063a. An example of the $\zeta$-factor determination is shown in Fig. 2(a): closed and open circles indicate experimentally determined $\zeta$-factors from the SRM2063a and $\zeta$-factors estimated from experimental $\zeta$-factors, respectively. To estimate the $\zeta$-factors from the experimental $\zeta$-factors, the detector efficiency parameters, such as thicknesses of ice and dead layers, by fitting. Therefore, the detector efficiency can also be determined in the $\zeta$-factor estimation, as shown in Fig. 2(b). These standard specimens especially in thin-film form for AEM are still limited or not available. Further development of such standard thin specimens is essential for superior X-ray analysis in AEM. 


\section{References}

[1] R.F. Egerton \& S.C. Cheng, Ultramicrosc. 55 (1994), 43.

[2] J.C. Bennett \& R.F.Egerton, J. Microsc. Soc. Am., 1 (1995), 143.

[3] K. Wong \& R.F. Egerton, Proc. Microscopy and Microanalysis 1995, Jones and Begell Publishing New York (1995), 600.

[4] C.E. Lyman, et al., J. Microsc. 176 (1994), 85.

[5] M. Watanabe, Microsc. Microana. 16 (2010), Suppl. 2, 260.

[6] M. Watanabe, (2009), XUtils - X-ray analysis utilities for Gatan DigitalMicrograph, available from Department of Materials Science \& Engineering, Lehigh Univeristy, Bethlehem, PA18015 (http://www.lehigh.edu/ maw3/research/xutilmain.html).

[7] E.B. Steel et al, Microsc. Microana. 3 (1997), Suppl. 2, 903.

[8] M. Watanabe and D.B. Williams, J. Microsc. 221 (2006), 89.

[9] The author (MW) wishes to acknowledge financial support from the NSF through grant DMR-0804528.

\section{Fig. 1 (a)}
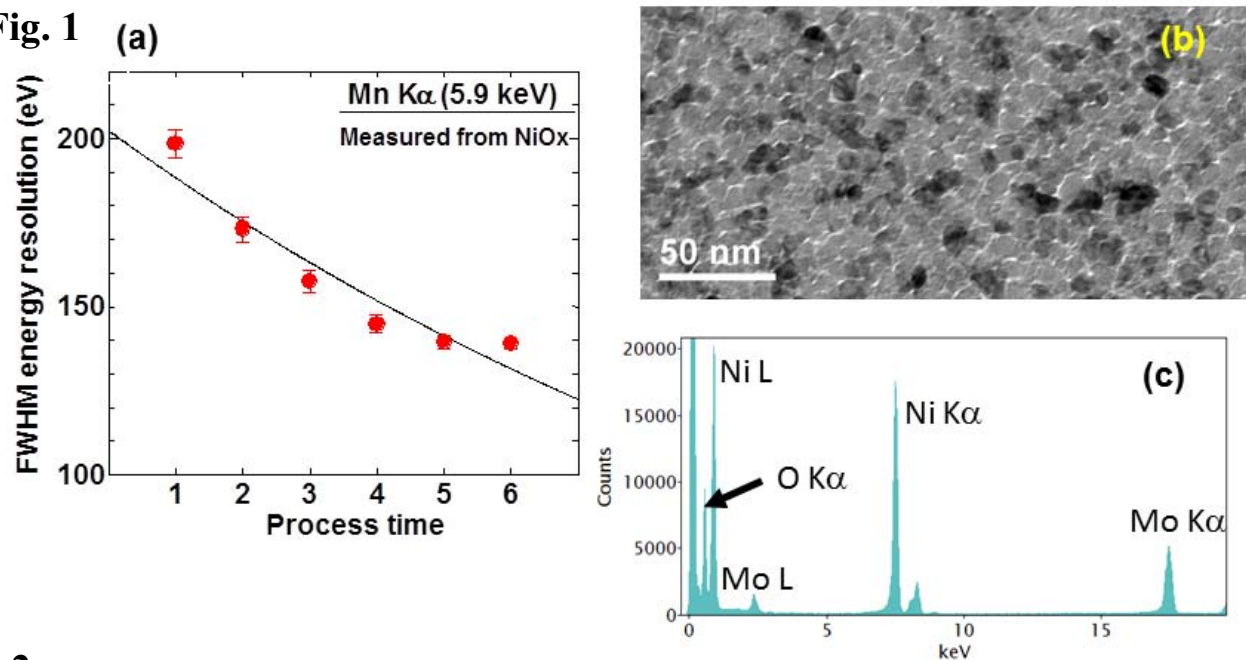

Fig. 2

(a)

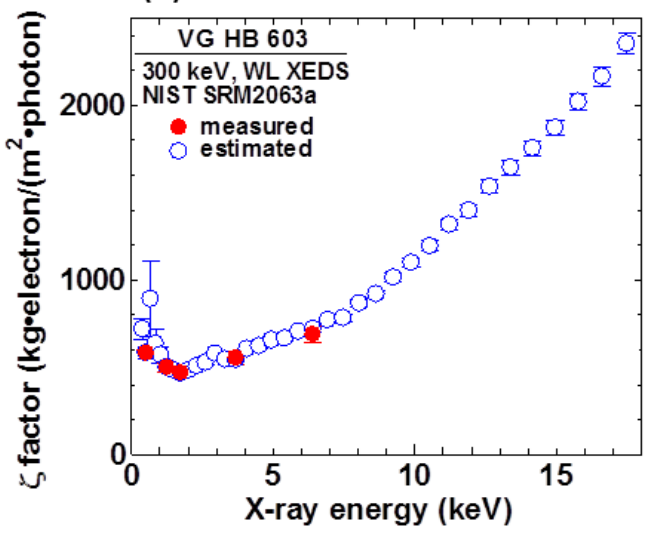

(b)

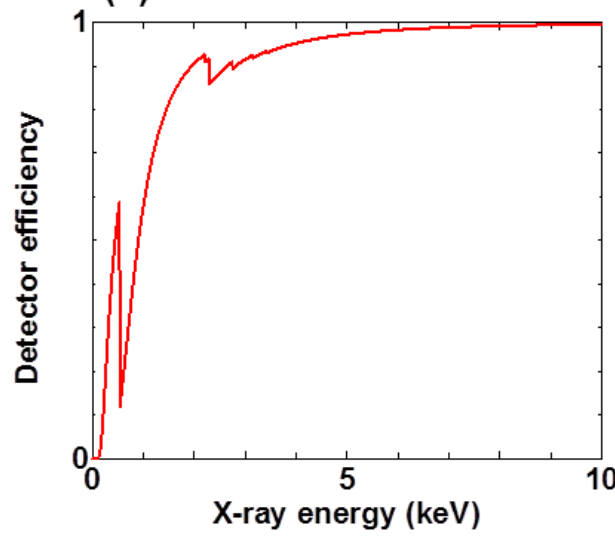

Figure 1: (a) System energy resolution determined from the NiOx test specimen. (b) microstructure and (c) an XEDS spectrum from a NiOxL fabricated at Lehigh.

Figure 2: (a) Determined and estimated $\zeta$-factors using the NIST SRM2063a thin film and (b) the detector efficiency determined after the $\zeta$-factor estimation [8]. 